\title{
Evaluación de las experiencias participativas en la gestión local de Cataluña: potencialidades y amenazas*
}

\author{
Carola Castella ${ }^{* * *}$ \\ Laia Jorba ${ }^{*{ }^{*} * *}$
}

Palabras clave: Evaluación, participación local, gobierno, gestión, innovación.

En los últimos años estamos siendo testigos de un intenso debate alrededor de la participación ciudadana en el ámbito local, debate paralelo al desarrollo de numerosas experiencias participativas alrededor del mundo. A nivel académico, la discusión se ha ido desplazando paulatinamente de la justificación de una participación política y el análisis de la práctica participativa -con el objetivo de sistematizar los requerimientos técnicos y prácticos para un desarrollo óptimo de los mecanismos participativos-, hasta la necesidad de una planificación y estructuración de la participación, por ejemplo, a través de planes estratégicos. Esta segunda etapa de desarrollo de la investigación en participación está asimismo llevando a una tercera, centrada en el estudio académico y práctico de los resultados de esos primeros años de experiencias participativas: en concreto, el objetivo es poder determinar la calidad del amplio abanico de prácticas participativas que se despliegan hoy día en el mundo local y, paralelamente, empezar a contrastar la verdad empírica de las promesas asociadas a la práctica participativa ${ }^{1}$. Así, la agenda futura de la investigación en participación se enfoca al desarrollo y contraste de los incipientes elementos y mecanismos de evaluación ${ }^{2}$, así como al establecimiento de medidas para determinar el impacto real en las políticas públicas.

En este artículo partimos de la identificación de criterios para el análisis de la calidad de la participación. Para ello se han escogido un conjunto de experiencias desarrolladas en distintos municipios catalanes a lo largo

"Este artículo es el resultado de un análisis más amplio de evaluación de los convenios y ayudas a entes municipales y supramunicipales para planes y fomento de la participación ciudadana otorgados por el Departamento de Relaciones Institucionales y Participación de la Generalitat de Cataluña.

Investigadora del Instituto de Gobierno y Políticas Públicas de la Universidad Autónoma de Barcelona.

*Investigadora del Instituto de Gobierno y Políticas Públicas y profesora asociada del Departamento de Ciencia Política y Derecho Público de la Universidad Autónoma de Barcelona.

${ }^{1}$ Entre estas promesas hallamos la mejora de los resultados de las políticas públicas, es decir, una mayor eficiencia, una mayor legitimidad de los resultados y las decisiones tomadas participativamente, y una mayor capacitación ciudadana a nivel individual y colectivo.

${ }^{2}$ Véase, por ejemplo, Anduiza y de Maya (2005), Font y Blanco (2003), Kaleidos (2006), Jorba, Martí y Parés (próxima publicación). 
del $2005^{3}$. Los proyectos elegidos son los solicitantes de una subvención del «programa de ayuda y promoción de experiencias de participación a entes locales» del Departamento de Relaciones Institucionales y Participación de la Generalitat de Cataluña (en adelante DGPC). En este sentido, además del análisis de la calidad de la participación, el estudio también permite una reflexión sobre la importancia y eficacia del apoyo de entes supramunicipales a la promoción de la participación local.

El artículo se estructura en dos apartados. En primer lugar, se analiza el perfil de los distintos entes locales solicitantes de la subvención. Así, un primer análisis extensivo, basado en la explotación de datos estadísticos nos permite conocer con mayor detalle cuales son los entes solicitantes. En segundo lugar, se hace un análisis en profundidad en función de unos criterios de calidad. Se han seleccionado 45 casos para realizar esta evaluación, selección en la que incluimos todos los convenios para la realización de planes de participación, y una selección representativa de 15 programas de participación ${ }^{4}$. Para el segundo nivel de análisis, se diseñaron cuestionarios específicos, complementando en algunos casos la información recibida de los responsables del proyecto con entrevistas personalizadas. A continuación presentamos las conclusiones del estudio, empezando por el análisis extensivo, para luego entrar en el detalle de los proyectos seleccionados.

\section{1. ¿Qué tipo de Administraciones locales solicitan y acceden a las ayudas? ¿Para qué temas?}

En este apartado nos centraremos en el perfil de los Ayuntamientos y Consejos Comarcales ${ }^{5}$ que solicitaron las ayudas en el 2005: ¿Cómo son estos entes?, ¿dónde se hallan?, ¿para qué temas piden ayudas?, etc. La DGPC otorgó ayudas al 40\% de las solicitudes presentadas en la convocatoria de 2005. Éstas cubrieron el $67 \%$ del importe solicitado por los entes municipales, después de que la DGPC hiciera un cálculo de los costes para eliminar subestimaciones y sobrestimaciones de los propios entes solicitantes.

Analizando las características del perfil de estas Administraciones locales se pueden destacar dos elementos importantes. El primero es la comprobación de que muchos proyectos presentados, y concedidos, se concentran en la demarcación de Barcelona ${ }^{6}$, a pesar de que la DGPC intentó compensar este hecho con subvenciones a entes que no se hallaban en esta demarcación. El segundo elemento, es la constatación de la diversidad temática de los proyectos, lo que nos lleva a pensar en el uso extensivo de las herramientas participativas para todo tipo de política pública. A continuación analizamos con más detalle estos dos elementos apuntando algunas de sus causas.

\subsection{Concentración de solicitudes en el entorno metropolitano y potenciación de otras zonas}

Atendiendo al territorio, se puede afirmar que las solicitudes se concentran en los municipios de la provincia de Barcelona (acumulan mayor número de solicitudes y, en muchos casos, un mismo ente local ha presentado más

\footnotetext{
${ }^{3}$ Actualmente se está realizando un análisis de las experiencias desarrolladas durante el 2006. Los datos de este segundo estudio son preliminares; aún así, y si se estima oportuno a lo largo del texto, se irán introduciendo elementos de contraste con el presente análisis. En el estudio del 2005, además de las autoras del presente artículo, también participó Joel Martí, profesor de Sociología de la UAB e investigador del IGOP.

${ }^{4}$ La selección se ha hecho en función del número de habitantes, la temática abordada, la composición política del gobierno, la ubicación territorial y la cuantía de la subvención otorgada finalmente.

${ }^{5}$ Un Consejo Comarcal es una Administración pública propia de Cataluña nacida en el marco de la Ley 6/1987 aprobada por el Parlamento de Cataluña, organización territorial prevista en el artículo 5 del Estatuto de Autonomía.

${ }^{6}$ Las demarcaciones son divisiones territoriales que agrupan distintos municipios, siendo un poco menores en cuanto a extensión y habitantes que las provincias. Existen, en total, siete demarcaciones en Cataluña: Barcelona, Cataluña central, Gerona, Lérida, Alto Pirineo y Arán, Tarragona y Tierras del Ebro.
} 
de una). Como se puede ver en la Tabla 1, los entes de la demarcación de Barcelona y Cataluña central concentran el 58,4\% del total de las 327 solicitudes. Las causas son diversas, pero señalamos las dos más importantes:

- En esta provincia hallamos muchos municipios con un elevado número de habitantes. Precisamente las poblaciones grandes son las que tienen mayores recursos propios para desarrollar iniciativas participativas y los que, por tanto, han sido pioneros en este tipo de proyectos en los últimos años; consecuentemente, son los más propensos a presentarse a la convocatoria.

- Además, el apoyo de Administraciones supramunicipales (en recursos económicos y formación) parece relevante y vital para que los Ayuntamientos medianos y pequeños puedan incorporarse a estas prácticas de gobierno. En el caso de la provincia de Barcelona, el Centro de Participación Ciudadana de la Diputación de Barcelona ha tenido un rol impulsor importante en los últimos años.

A pesar de la afirmación anterior, la extensión y diversidad de los entes solicitantes indica que también están representados el resto de demarcaciones y los municipios más pequeños. De hecho, no deja de ser significativo que casi un 12\% de las solicitudes correspondan a pueblos con menos de 1.000 habitantes. Hay dos elementos que contribuyen a explicarlo:

- Los criterios de otorgamiento de ayudas utilizados por la DGPC han sobreponderado los proyectos de municipios pequeños y también de fuera de la demarcación de Barcelona y Cataluña central (ya que en éstas existían, simultáneamente, una convocatoria de ayudas de la Diputación de Barcelona).

- La tarea de difusión presencial de la convocatoria que hizo la DGPC ha tenido sus frutos dado que muchos entes han presentado solicitudes para realizar este tipo de proyectos por primera vez.

\section{TABLA 1}

Entes según demarcación. Comparación entre proyectos solicitados y otorgados

\begin{tabular}{|l|c|c|c|c|c|}
\hline & \multirow{2}{*}{$\begin{array}{c}\text { Municipios } \\
\text { Cataluña }\end{array}$} & $\begin{array}{c}\text { Proyectos } \\
\text { solicitados }\end{array}$ & $\begin{array}{c}|c| \\
\text { \% sobre } \\
\text { proyectos }\end{array}$ & $\begin{array}{c}\text { \% sobre total } \\
\text { otorgada }\end{array}$ & Mediana $€$ \\
\hline Barcelona & 17,4 & 43,4 & 35,3 & 41,3 & $7.415 €$ \\
\hline Cataluña central & 19,3 & 15,9 & 14,4 & 12,0 & $5.289 €$ \\
\hline Gerona & 22,0 & 18,7 & 24,8 & 22,5 & $5.752 €$ \\
\hline Lérida & 15,7 & 5,2 & 4,6 & 5,1 & $7.003 €$ \\
\hline Alto Pirineo y Arán & 6,4 & 4,3 & 4,6 & 4,3 & $5.989 €$ \\
\hline Tarragona & 13,8 & 8,0 & 10,5 & 10,0 & $6.054 €$ \\
\hline Tierras del Ebro & 5,6 & 4,6 & 5,9 & 4,8 & $5.203 €$ \\
\hline Total & $100 \%(947)$ & $100 \%(327)$ & $100 \%(153)$ & $970.000 €$ & $\mathbf{6 . 3 4 0} €$ \\
\hline
\end{tabular}

Fuente: Elaboración propia. 


\subsection{Diversidad temática, valoración de los planes de participación y predominio de proyectos urbanísticos}

Para analizar los temas abordados en los proyectos, es necesario, en primer lugar, distinguir entre «planes de participación» y «programas participativos», diferenciación que establecía la misma DGPC?:

a) Planes de participación. Es necesario destacar el escaso número de solicitudes presentadas bajo este epígrafe donde, proporcionalmente, se han otorgado muchas más ayudas. Se han subvencionado un $76 \%$ de las solicitudes de planes, en contraste con el $43 \%$ de los programas de participación. ¿Quién presenta planes de participación? En general (con algunas excepciones), se trata de municipios grandes, de la demarcación de Barcelona y, sobre todo, con experiencia en participación. Parece que la necesidad de elaborar planes de participación se plantea, especialmente, en aquellos municipios en los que ya existen órganos estables y procesos participativos en marcha, en los que posteriormente se ve la necesidad de reordenar globalmente la estrategia de participación. Aún así, hay municipios de otras demarcaciones que, sin experiencia previa, han desarrollado algún plan estratégico, siendo éstos minoritarios.

b) Programas de apoyo a la participación. En este caso, el tipo de municipio y las temáticas son mucho más variadas, como se aprecia en la Tabla 2. Una parte importante tiene, como temática central, la propia «participación» (apoyo al tejido asociativo, etc.); destacan también los proyectos urbanísticos- un ámbito que es central en la agenda política local, siendo en algunos casos la participación obligatoria, como en las revisiones de los planes urbanísticos; finalmente, también hallamos proyectos con temáticas relativas a la convivencia, mediación e inmigración. Asimismo, el tipo de proyectos que han recibido ayudas más elevadas son los clasificados bajo el epígrafe «planes estratégicos y presupuestos participativos» y «participación y asociacionismo». Teniendo en cuenta el perfil de los municipios, no se puede afirmar que determinadas temáticas se concentren en zonas concretas o poblaciones de determinadas dimensiones.

\section{TABLA 2}

Proyectos solicitados y otorgados según descriptor

\begin{tabular}{|c|c|c|}
\hline & $\begin{array}{l}\text { Proyectos } \\
\text { solicitados }\end{array}$ & $\begin{array}{l}\text { Proyectos } \\
\text { otorgados }\end{array}$ \\
\hline Participación y asociacionismo & 36,6 & $39,9 \%$ \\
\hline Urbanismo & $15,5 \%$ & $17,0 \%$ \\
\hline Convivencia, mediación e inmigración & $9,2 \%$ & $11,8 \%$ \\
\hline Nuevas tecnologías & $9,8 \%$ & $9,8 \%$ \\
\hline Planificación estratégica y presupuestos participativos & $5,2 \%$ & $5,9 \%$ \\
\hline A21 y sostenibilidad & $3,7 \%$ & $5,2 \%$ \\
\hline Otros & $22,0 \%$ & $10,5 \%$ \\
\hline Total & $100 \%(327)$ & $100 \%(153)$ \\
\hline
\end{tabular}

Fuente: Elaboración propia.

${ }^{7}$ Los planes tienen una vocación mucho más estratégica y de planificación general de la participación en el municipio, mientras que los programas son más acotados tanto por lo que se refiere a la temática, como a los objetivos y duración. 


\section{La calidad de los procesos participativos: proceso y resultados}

Tal y como comentamos previamente, a partir de todos los proyectos subvencionados se ha realizado un análisis detallado del conjunto de planes de participación y de 15 programas de apoyo a la participación, seleccionados con criterios de representatividad del conjunto de entes solicitantes.

\section{1. ¿Qué se ha analizado en cada caso? Modelo teórico}

El modelo de análisis para la evaluación de los proyectos pone en relación determinados factores explicativos -como el perfil del municipio, el contexto de la participación y el tipo de proyecto realizado-, con dos dimensiones que, desde las teorías existentes sobre participación, consideramos clave: el proceso de realización de la experiencia, que se desarrolla participativamente (Rebollo y Martí 2002), y los resultados de la misma.

Estas dos dimensiones se ven también influidas por diferentes factores, de los cuales hemos tenido en cuenta los más relevantes para nuestro estudio, a saber: a) el perfil del municipio; b) el contexto o circunstancias del municipio en relación a la participación; c) y, finalmente, el tipo de proyecto realizado. Esquemáticamente, el modelo de análisis incorpora los siguientes elementos o variables independientes, factores explicativos, y variables dependientes, factores a explicar.

\section{- Factores explicativos}

a) El perfil de los municipios. Aquí es importante plantearse qué tipo de entes locales acaba consolidando estrategias de participación, asimismo como preguntarse si las características sociodemográficas tienen alguna influencia en el momento de determinar objetivos y diseños participativos. Para todas estas cuestiones cabe tener en cuenta la dimensión del municipio (en dispersión territorial y en población), su situación geográfica, así como distinguir entre las Administraciones municipales y las supramunicipales, dado que no es lo mismo impulsar estrategias participativas en municipios pequeños que en poblaciones que superen ciertas dimensiones.

b) El contexto. Un elemento importante de contexto que puede influenciar en el diseño y desarrollo de la participación es la tradición existente en estos temas, así como la existencia de los recursos necesarios -humanos, técnicos, económicos y temporales-, internos y/o externos, para poder desarrollar el proyecto. También se ha analizado hasta qué punto la existencia de áreas y estructuras consolidadas internas de participación permite un mejor desarrollo del proceso y el cumplimiento de los objetivos planteados en el proyecto.

c) Tipo de proyectos. Se han analizado los proyectos en función de la divisoria formal que establecía la misma convocatoria (convenios para el desarrollo de planes de participación y programas de apoyo a la participación). ¿Hasta qué punto esta tipología determina el desarrollo y el cumplimiento de los objetivos? Y si esto se da, ¿cuáles pueden ser las causas de estas diferencias? También se analizan las temáticas tratadas y los principales objetivos que se buscan en los diferentes procesos participativos. 


\section{- Dimensiones analizadas}

Las dos dimensiones analizadas, el proceso y los resultados, se concretan en criterios más específicos, criterios que son flexibles dado que la realidad sociodemográfica, política y organizativa de los municipios y las comarcas es diferente, así como la naturaleza y objetivos del proyecto. En primer lugar, los criterios de análisis del proceso de realización que se suelen considerar son diversos, pero se pueden sintetizar en los siguientes:

a) Objetivos, contenidos y adecuación de los resultados a los objetivos iniciales. El planteamiento de objetivos claros, para los gestores del proyecto y para los propios participantes, es una de las características más importantes de cualquier proceso: dota de coherencia al proyecto, aportando una línea clara de trabajo y limitando tanto lo que se quiere conseguir como lo que no se puede conseguir. Este último elemento es imprescindible para no frustrar las expectativas de los participantes y eliminar las demandas excesivas que pueden surgir en procesos ambiguos. De este modo, se ha analizado no solamente el tipo de objetivos que los proyectos se han planteado, sino también en qué grado éstos se han cumplido, hasta qué punto han guiado el desarrollo del proceso y, en caso de no obtener los resultados esperados, cuáles han sido las causas que no lo han permitido, puntos sobre los que podrá incidirse en el futuro.

b) Iniciativa y gestión del proceso. ¿Quién toma la iniciativa del proceso participativo? ¿Con qué objetivos? Partiendo de proyectos impulsados desde Gobiernos locales, las iniciativas no provienen siempre de los mismos actores: pueden, por ejemplo, provenir de alcaldía, con acuerdo e implicación de todo el pleno municipal; o bien pueden estar impulsadas desde una sola área; y, en este segundo caso, ésta puede contar con la complicidad y implicación de otras, o no. En este aspecto, consideramos importante poner en relación los objetivos del proyecto con el actor que lo promueve, y el grado de corresponsabilización y/o implicación de diferentes áreas políticas, técnicas y administrativas que sean pertinentes en cada caso. La situación ideal, la que permite un mejor desarrollo de los procesos, se produce cuando hay consenso a nivel interno o político, entre los miembros del equipo de gobierno y entre estos y la oposición, pero también a nivel externo o social, con otros actores del proceso. En estos casos, no es necesaria una unanimidad referente a los resultados del proceso, sino la predisposición o voluntad al diálogo y unas normas de desarrollo del proceso ampliamente aceptadas y/o susceptibles de ser negociadas a lo largo de éste. Asimismo, cuando el trabajo interno es transversal, es decir, hay una labor conjunta ya no sólo a nivel político, sino también a nivel técnico y administrativo, hay una mayor garantía de fluidez y eficiencia de los procesos y menos resistencias a implementar los resultados (Font y Blanco 2003). A pesar de que teóricamente los procesos participativos son por definición transversales (ya que, por un lado, tocan temáticas que suelen afectar a otras áreas o departamentos que no sean participación; y, por el otro, los resultados de los procesos participativos frecuentemente tienen que implementarse a partir de otras áreas o departamentos), a menudo esta transversalidad es testimonial o inexistente. ¿Hasta qué punto existe pues una transversalidad real? Otro elemento a considerar es la existencia de otros grupos, empresas o entidades que puedan facilitar el desarrollo de los procesos participativos. En este aspecto, se puede considerar, por un lado, la existencia de un grupo promotor del proyecto que vele por las «coherencias» del proceso; y por el otro, la existencia de empresas/entidades o técnicos profesionales externos que pueden facilitar el desarrollo de los proyectos de participación.

c) Los participantes. Font y Blanco (2003) hacen referencia a la extensión, pluralidad y representatividad de los participantes. No se trata únicamente de una cuestión numérica o extensión de la participación sino, también, de la presencia de una diversidad o pluralidad de perfiles, tanto sociodemográficos (edad, género, minoría étnica, etc.) como de posicionamiento o puntos de vista respecto a la temática tratada y el papel que estos participantes tienen en las diferentes fases del proyecto. ¿Se han creado estrategias 
de movilización específicas para atraer perfiles de participantes que habitualmente no han estado presentes en procesos previos? ¿Se distingue entre ciudadanos y entidades? ¿Qué consecuencias tiene esto en el diseño del proceso?

d) Diseño y encaje del proceso en el sistema participativo. Aquí se pueden considerar muchos factores, ya que se trata de un elemento central para el desarrollo del proyecto y que hace referencia, principalmente, a la forma que este acaba tomando. Podemos identificar los siguientes elementos:

- Diseño de la estructura. El proceso participativo está marcado por los diferentes espacios y contenidos de información/comunicación. Estos espacios deben garantizar que los participantes puedan expresarse con conocimiento de causa y con libertad. Por este motivo es necesaria una información plural y que los objetivos estén definidos de forma transparente y clara.

- Intensidad de la participación. Arnstein (1971) definió la intensidad en función del rol que tienen los ciudadanos y las asociaciones en los diferentes espacios creados para la participación. El autor distingue entre los siguientes niveles de participación, niveles que son acumulativos (cada uno engloba los anteriores): información, comunicación, consulta, deliberación y participación en la toma de decisiones. En cada uno de los niveles se requiere, progresivamente, una mayor implicación y menor pasividad de los participantes. Especialmente importante es la existencia de debate y deliberación en los procesos ya que puede cambiar la opinión de la ciudadanía sobre los aspectos sometidos a participación y promover las disposiciones personales adecuadas hacia los procesos democráticos en sentido amplio.

- La metodología y el funcionamiento de los espacios de deliberación pueden influir bastante en el desarrollo y los resultados de un proceso participativo y de toma de decisiones, ya se trate de estructuras ad boc (creadas específicamente para el proceso participativo) o estructuras existentes (Consejos Consultivos, forums estables, etc.). Especialmente importante es analizar las dinámicas de debate/comunicación, identificando el uso de herramientas que permitan compensar las posibles desigualdades de partida (de información, de capital cultural, de capacidad argumentativa, etc.) de los participantes (por ejemplo, Delbecq 1984).

- Sistemas de participación. Con frecuencia surgen dificultades, contradicciones y solapamientos por la copresencia de diferentes proyectos paralelos al analizado; es el caso de aquellas ciudades que, por ejemplo, cuentan con proyectos locales e impulsan un proceso participativo para definir el Plan Estratégico de Ciudad. ¿Cómo se integran todos estos procesos? ¿Qué grado de coherencia tiene la iniciativa con los anteriores proyectos? Se trata de analizar la integración del proyecto de participación en el conjunto de la acción de gobierno (Subirats et al. 2001), para garantizar su sostenibilidad en el tiempo y la retroalimentación que redunde en una mayor eficiencia general. Además si, un proceso participativo se encaja en un sistema de participación más amplio y complejo, hay que preguntarse: ¿Qué grado de relación existe entre el proceso participativo analizado y el sistema estable de participación (reglamentos, Consejos Consultivos Sectoriales y/o Territoriales, webs interactivas, etc.) que funciona de forma regular?

- Fases del proceso. En último lugar, se han tenido en cuenta las diferentes fases de las que consta un proceso. Cada una de estas fases tiene requisitos diferentes y, por tanto, se tendrá que modificar el diseño y la forma que toma la participación en cada una de ellas ¿Se adaptan efectivamente los diseños según las fases? ¿Tiene esto consecuencias para los resultados del proceso? 
La segunda dimensión a analizar, a parte del mismo proceso de participación, son los resultados de éste. No nos estamos refiriendo a los efectos o el impacto de la política pública que surja del proceso de participación, algo que requeriría un análisis a medio o largo plazo, sino los resultados que ha producido el mismo proceso:

a) Resultados sustantivos. Una experiencia de participación local, al menos en los términos que aquí se están abordando, debería desembocar en un plan, programa o proyecto que contemple determinadas líneas de actuación y acciones referidas a la temática o área que se está tratando; ya sea vinculado a determinados colectivos de población (jóvenes, tercera edad, inmigración, etc.), o a sectores de intervención (urbanismo, cultura, medio ambiente, etc.). Estos resultados pueden contener, también, acciones referidas a la mejora de la organización local o de la capacitación de la ciudadanía.

b) Elementos relacionales. Ya que la participación implica vínculos entre las entidades, ciudadanos y la organización municipal/supramunicipal, es pertinente analizar los cambios que se han producido a lo largo del proceso de participación. Estos aspectos contribuyen, en última instancia, a la generación de una cultura cívica y a lo que algunos autores designan como «capital social» (Putnam 1993).

Finalmente, la evaluación es otro aspecto que se ha tenido en cuenta en el momento de analizar los proyectos subvencionados, no tanto por tratarse de una dimensión distintiva al proceso y a los resultados, sino porque la consideramos de vital importancia para mejorar los procesos y aumentar la calidad de la participación. En este sentido, se ha analizado si el proyecto preveía y/o realizó algún sistema de evaluación interna y cuales eran sus características, considerando especialmente si ésta era interna o externa y participada o no participada.

Sobre esta aproximación teórica de factores explicativos y dimensiones, se definió un modelo de análisis común que se fue ajustando para cada uno de los proyectos en función de sus objetivos, metodologías y temáticas.

\subsection{Análisis de los resultados de los casos seleccionados}

\subsubsection{Objetivos, contenidos y adecuación de los resultados a los objetivos iniciales}

Se puede hacer una primera distinción formal entre los programas de apoyo a la participación (financiados vía subvención) y los planes de participación (financiados vía convenio) ${ }^{8}$. Los programas, las bases de cuya convocatoria eran muy abiertas, comprendían procesos y mecanismos participativos muy diversos, de temática variable (urbanismo, mediación, asociacionismo, etc.) y con objetivos de abasto diverso. Entre los programas analizados, también encontramos algunos planes participativos.

Por otro lado, los planes de participación se orientan, en general, al diseño, desarrollo e implementación de unas líneas estratégicas para abordar la participación ciudadana. Podemos diferenciar a grandes rasgos cuatro modelos en relación a los objetivos y contenidos de los proyectos: 1) Modelo «integral»: incluye una fase de diagnosis y una prepositiva, en el ámbito de la organización municipal y en el de la ciudadanía. 2) Modelo «ordenación de canales y órganos»: se trata de recoger, inventariar, describir, y hasta, analizar los canales y órganos de participación existentes en la población. 3) Modelo «reglamento de participación»: el plan de participación se focaliza en la elaboración o implementación del reglamento de participación ciudadana. 4) Modelo de «complemento»: se vincula la realización del plan de participación a otros proyectos que están en funcionamiento en el municipio.

\footnotetext{
${ }^{8}$ En la Tabla 3 puede verse la distribución de proyectos seleccionados para el análisis en función del tipo de proyecto.
} 


\section{TABLA 3}

Tipo de proyectos analizados (planes y programas de participación)

\begin{tabular}{|l|l|c|}
\hline \multicolumn{1}{|c|}{} & & Frecuencia \\
\hline Planes de & Complemento & 4 \\
& Integral & 15 \\
\hline \multirow{5}{*}{ Programas } & Ordenación & 6 \\
& Reglamento & 2 \\
\hline & Diversos & 2 \\
& Mediación & 1 \\
& Planes de participación y estratégicos & 4 \\
& Presupuestos Participativos & 1 \\
& Urbanístico & 7 \\
\hline & Total & 44 \\
\hline
\end{tabular}

Fuente: Elaboración propia.

En general, estos modelos de planes de participación responden también a diferentes perfiles de municipio y comarca que se encuentran en diferentes momentos participativos. ¿Cuando se desarrolla un plan de participación?

En primer lugar, hallamos una serie de municipios que, después de iniciar en los últimos años experiencias de participación, constatan la necesidad de una reflexión global que ordene los canales e iniciativas existentes con el objetivo de definir estrategias futuras de participación. La mayoría de estos municipios tienen ya reglamentos de participación ciudadana y una estructura de Consejos Sectoriales estables en funcionamiento.

En segundo lugar, municipios sin experiencias anteriores y frecuentemente sin reglamentos de participación que, atraídos por el nuevo contexto propicio, quieren desarrollar estrategias municipales globales mediante planes de participación. En estos casos, el plan puede significar una oportunidad para iniciar dinámicas participativas allí dónde, hasta el momento, no se había impulsado ninguna. En general, estos municipios valoran a posteriori empezar por experiencias de temática y temporalidad acotada, ya que parece que estos procesos se puedan gestionar y controlar mejor con los recursos cognitivos, técnicos y económicos de qué disponen.

En tercer lugar, municipios que, en base a una diagnosis de la realidad participativa existente, elaboran un reglamento de participación ciudadana. Son muy pocos Ayuntamientos, y en estos casos se ha hecho con un nivel de participación muy bajo.

Finalmente, también encontramos otra diferencia entre los proyectos presentados como planes de participación, específicamente en relación a los espacios de reflexión e intervención:

Algunos planes de participación diferencian claramente entre una fase de reflexión e intervención interna, es decir, entre una fase centrada en la organización del Ayuntamiento respecto la participación (comunicación, coordinación y transversalidad en los niveles político y técnico) y una fase de reflexión e intervención externa, centrada en las estrategias y formas de relación con la ciudadanía. Esta distinción se debe principalmente a los requisitos estables y definidos en la convocatoria de la DGPC. En muchas de las experiencias analizadas, el desarrollo del proyecto subvencionado ha cubierto únicamente la primera fase de reflexión interna. 
Otros planes de participación, en cambio, no distinguen entre los dos niveles de trabajo y se centran, sobre todo, en la «dimensión externa» del plan; es decir, tratan de definir, poner en funcionamiento o revisar los mecanismos de participación ciudadana, entre los que también incluyen campanas de comunicación y difusión.

Otro elemento a valorar para el conjunto de los proyectos es la adecuación de los objetivos realizados con los presentados inicialmente. Este elemento es variable y desigual según los proyectos:

Por un lado, tenemos las experiencias que han desarrollado los objetivos previstos. En general se trata de municipios que tienen un bagaje y una tradición participativa importante. Acostumbran a ser proyectos que ya estaban en funcionamiento en el momento de recibir la subvención, por tanto con una duración superior a los plazos de las ayudas (entre dos-tres años), y que prevén que la subvención cubra únicamente una parte. La existencia de acuerdos políticos para realizarlos (hecho que se da, normalmente, cuando hay empresas externas que gestionan el proceso) y el hecho de tener otra subvención para el mismo proyecto son también hechos que favorecen el cumplimiento de los objetivos.

En segundo lugar, están aquellos proyectos que han desarrollado parcialmente los objetivos. En este caso, la falta de experiencia previa y de formación, a nivel político y técnico, ha sido un factor restrictivo importante, así como, en algunos casos, la falta de recursos propios. Además, la llegada tardía de la subvención ha retrasado el inicio de muchos procesos y, por tanto, en el momento del análisis no se habían finalizado.

En tercer lugar, están aquellos municipios que han revisado y adaptado significativamente los objetivos una vez iniciado el proceso. Los motivos pueden ser paralelos a los de los casos anteriores; pero también se han modificado por motivos políticos (por ejemplo, el conflicto entre miembros de un Gobierno de coalición o entre el equipo de Gobierno y la oposición).

Finalmente, hay dos casos que han renunciado a la subvención.

\subsubsection{Iniciativa, gestión y organización institucional del servicio de participación ciudadana}

En la mayoría de casos la iniciativa del proyecto corresponde al área o servicio de participación ciudadana con un porcentaje del 48,6\%, depende de la regiduría de participación o de alguna otra. En Consejos Comarcales y Ayuntamientos pequeños, los cuales no suelen disponer de servicio de participación ciudadana, la iniciativa políica corresponde normalmente a la presidencia o a la alcaldía, excepto en aquellos casos en que se trata de temas específicos (urbanismo, mediación...).

En el caso de los planes de participación, y por su carácter global, es importante que, independientemente del área desde donde se conduzca el proceso, éste cuente con el apoyo y corresponsabilización de todas las áreas municipales. Según la documentación aportada por los Ayuntamientos, parece que esto es así en la mayoría de los casos, a pesar de los diferentes grados que hemos detectado:

Inicialmente, hay casos en que la transversalidad es sobre todo interna a la regiduría, es decir, el/la regidor/a y el/la técnico/a de «participación» lo son también de otras áreas (por ejemplo, de bienestar social, medio ambiente, comunicación o educación), hecho que automáticamente facilita que los proyectos participativos aborden en muchos casos temáticas que son competencias del regidor de participación. 
Posteriormente, frecuentemente se da una transversalidad parcial, es decir, hay contacto, colaboración y comunicación entre diferentes áreas municipales (a nivel político y/o técnico) en alguna fase del proyecto (por ejemplo, en el desarrollo de un taller concreto en el que participan distintos técnicos).

Por último, están aquellos casos (muy minoritarios) en que la transversalidad se da a un alto nivel, es decir, se trabaja conjuntamente de forma constante entre diferentes áreas a lo largo del proceso.

En general, como se ha comprobado a través de las entrevistas, se tiende a sobreestimar la transversalidad real, sea ésta total o parcial. Tampoco se diferencia entre los niveles de intensidad de participación.

A pesar de que, como se observa en la Tabla 4, un $73 \%$ de los entes locales analizados dicen trabajar transversalmente, esta realidad no se ha corroborado a través de las entrevistas ${ }^{9}$. Asimismo, se puede ver como se da una mayor tasa de transversalidad en los planes de participación. En los programas, a pesar de que en la mayoría de casos no hay transversalidad, cuando existe suele ser de carácter interno a la regiduría de participación ciudadana o de carácter global y puntual debido a la colaboración de proyectos participativos temáticos iniciados desde otras regidurías.

\section{TABLA 4}

Relación entre planes, programas y transversalidad

\begin{tabular}{|c|c|l|c|c|c|}
\hline \multicolumn{2}{|c|}{} & Plan & Programa & Total \\
\hline \multirow{2}{*}{ No } & Frecuencia & 5 & 5 & 10 \\
& Porcentaje & $17,9 \%$ & $55,6 \%$ & $27,0 \%$ \\
& \multirow{2}{*}{ Si } & Frecuencia & 23 & 4 & 27 \\
& Porcentaje & $82,1 \%$ & $44,4 \%$ & $73,0 \%$ \\
\hline \multirow{3}{*}{ Total } & Número & 28 & 9 & 37 \\
& Porcentaje & $100,0 \%$ & $100,0 \%$ & $100,0 \%$ \\
\hline
\end{tabular}

Fuente: Elaboración propia.

Por otro lado, y siguiendo a Font y Blanco (2003), el hecho de «que el proceso participativo se apoye en el acuerdo del conjunto de fuerzas políticas, aumenta mucho las posibilidades de que el conjunto del proceso y sus resultados sean aceptados ampliamente». En general, parece que este aspecto no se ha abordado específicamente en los proyectos analizados. La presencia de una empresa o entidad externa puede, en algunos casos, potenciar el acuerdo político, tanto a nivel del propio Gobierno como con la oposición.

Respecto a los recursos -bumanos y económicos-, internos y/o externos y la calidad de los proyectos, se pueden destacar unos cuantos elementos. En primer lugar, la disponibilidad de recursos económicos: en general, se da una relación positiva entre la disponibilidad de recursos y las posibilidades de realización de los objetivos proyec-

${ }^{9}$ Los datos de la tabla derivan de las respuestas del cuestionario de evaluación. La pregunta era general, no distinguía entre niveles e intensidades, lo que no permite valorar directamente si efectivamente se trabaja transversalmente o no y si el proyecto de participación ha contribuido a ello. 
tados. A pesar de ello, a los recursos económicos se le suman otros factores que pueden favorecer la calidad y cumplimiento de los objetivos, dándose normalmente una concentración de recursos de diferente tipo en un mismo proyecto, como los que analizamos a continuación ${ }^{10}$. Por ejemplo, también se da una relación positiva entre la existencia de un área o servicio de participación ciudadana, esté consolidado o no, y la calidad del proceso realizado y/o cumplimiento de los objetivos previstos, independientemente de quien lidere políticamente el proceso. Además, la contratación de una empresa o entidad externa, pero sobre todo la presencia de algín profesional de la participación para la gestión del proyecto, suele ser una garantía de calidad y finalización de los proyectos, incluso en mayor medida cuando trabajan conjuntamente con los técnicos y políticos del consistorio. En este sentido, los proyectos gestionados por personal externo suelen partir de una definición y planificación mucho más precisa y detallada, algo que aumenta potencialmente las posibilidades de éxito. En cambio, no se ha podido corroborar si el hecho de tener personal técnico propio a tiempo completo o parcial, es o no relevante, dado que en los cuestionarios no se ha reflejado esta distinción y en muchos casos se ha interpretado incorrectamente por parte del responsable del proyecto a analizar.

Es necesario, en última instancia, destacar la especificidad de las iniciativas supramunicipales (dos Consejos Comarcales), que presentan diferentes peculiaridades respecto al resto de proyectos analizados. En primer lugar, los dos casos analizados no cuentan con áreas de participación específicas, correspondiendo las iniciativas políticas de los proyectos normalmente a presidencia u otras áreas. Finalmente, las temáticas tratadas son de interés generales y de alcance supramunicipal.

\subsubsection{Participantes}

En los procesos participativos analizados se ha valorado positivamente la implicación tanto de la ciudadanía no asociada, como la participación de las entidades del municipio, siempre teniendo en cuenta la población objetivo de los proyectos.

De los programas y planes de participación analizados, tres cuartas partes, tal y como se aprecia en la Tabla 5, han abierto espacios de participación a entidades. La mayoría de estas asociaciones son agrupaciones de vecinos, culturales y medioambientales. Esta participación se da a menudo a través de los Consejos Sectoriales municipales. También hay casos, en que la participación, vehiculada a través de talleres y jornadas de debate, se dirige al conjunto de las asociaciones registradas en el municipio. En muy pocos casos, detectados a través de las entrevistas, el debate en el que participaban los representantes de las asociaciones se ha hecho extensivo a nivel interno, entre los miembros de las propias asociaciones.

Por lo que atañe a la participación individual, se puede destacar que la característica más generalizada de los programas y planes de participación analizados es la escasa presencia de ciudadanía no asociada. La participación individual tiene tan sólo un impacto significativo cuando se han diseñado estrategias específicas en esta dirección; y esta es, precisamente, una situación minoritaria. Además, no todas las estrategias que se han utilizado son propiamente «mecanismos de participación», puesto que se limitan a menudo a diseño informativo y de consulta. Entre estas estrategias encontramos, por ejemplo: sondeos de opinión, encuestas, entrevistas, grupos de discusión, forum electrónicos, etc. Paralelamente, hay confusión respecto a lo que se entiende por participación individual, mezclándose a menudo no sólo los mismos participantes en distintas fases del proyecto -algo que dificulta la calificación de la intensidad de la participación en éstas-, sino también con participantes de procesos paralelos.

\footnotetext{
${ }^{10}$ Sin embargo, en algunos casos una escasez de recursos puede eliminar incentivar el desarrollo de procesos creativos e innovadores, como se ha dado en alguno de los casos analizados.
} 


\section{TABLA 5}

Participación de asociaciones e individual en los proyectos analizados

\begin{tabular}{|l|c|c|c|c|}
\hline & \multicolumn{2}{|c|}{ Participación de } & \multicolumn{2}{c|}{ Participación } \\
\hline & Frecuencia & Porcentaje & Frecuencia & Porcentaje \\
\hline No & 9 & 24,3 & 19 & 51,4 \\
\hline Sí & 28 & 75,7 & 18 & 48,6 \\
\hline Total & 37 & 100,0 & 37 & 100,0 \\
\hline
\end{tabular}

Fuente: Elaboración propia.

Cuantitativamente, y en proporción al total de población, la participación es mucho más alta en los pequeños municipios, donde el esfuerzo de convocatoria es, lógicamente, menor y la proximidad entre las personas facilita que los procesos participativos tengan más poder de atracción. A pesar de ello, muchos municipios pequeños no han aprovechado este potencial participativo. En los municipios mayores (de más de 100.000 habitantes), encontramos menos estrategias para atraer la participación individual e, incluso, la participación de las asociaciones se acostumbra a limitar a las de segundo grado o agrupaciones de asociaciones.

Prácticamente no bay información sobre perfiles de participantes en ninguno de los proyectos, algo que dificulta un abordaje específico de la participación de individuos y grupos minoritarios. Tampoco hay casi presencia de grupos promotores de individuos y/o asociaciones que trabajen conjuntamente con el Ayuntamiento en el diseño y puesta en marcha de los procesos participativos. Los procesos están muy dirigidos desde los Ayuntamientos, sin una cogestión con asociaciones o individuos externos al consistorio.

En síntesis, se detecta la necesidad de definir: 1) Estrategias específicas para promover la participación de la ciudadanía no asociada. 2) Estrategias de control de la diversidad de los participantes. 3) Estrategias para no recurrir sólo a los «interlocutores habituales» (representantes de asociaciones) y aquellas personas que voluntariamente usan los mecanismos disponibles (forum, talleres). Es muy poco frecuente la selección específica de perfiles.

\subsubsection{Diseño y coordinación del proceso en el sistema participativo}

a) Diseño de la estructura e intensidad de la participación. ¿Cuáles son los espacios dónde se desarrolla la participación y qué tipo de actores se encuentra en estos espacios? En el caso de los planes de participación, los espacios más activos y/o estables han sido generalmente los que dependían directa y orgánicamente del propio Ayuntamiento, vehiculándose a través de los Consejos Sectoriales (espacios de participación y deliberación mixta), donde se da la mayor intensidad participativa. Por otro lado, los espacios abiertos a las asociaciones y al resto de la ciudadanía han sido, en general, puntuales (por ejemplo a través de talleres de debate) y convocados para realizar diagnosis, o para contrastar diagnosis técnicas previas y elaborar propuestas a partir de éstas.

En el caso de los programas de apoyo a la participación, la participación ba sido más integral, creando espacios de trabajo mixto en los diferentes momentos de los procesos participativos y con diferentes intensidades. Por ejemplo, los espacios de más intensidad participativa, que integran deliberación, toma de decisiones e incluso el seguimiento en la implementación de las propuestas aprobadas, tienen en común: incluir tanto representan- 
tes políticos y técnicos, como también miembros de asociaciones del municipio y, a veces, incluso ciudadanos a título individual; reunirse periódicamente; y, deliberar y evaluar propuestas como función principal. Difícilmente, la participación usa espacios de trabajo mixto más puntuales, como por ejemplo a través de talleres sectoriales.

Dejando de lado el espacio concreto en el que se desarrolla la participación, y siguiendo la gradación de la escala de la intensidad de la participación (información, comunicación, consulta, deliberación y toma de decisiones) propuesta por Arnstein (1971), se puede decir que, la gran mayoría de programas y planes han llevado a cabo una participación de carácter deliberativo y de toma de decisiones ${ }^{11}$, pero muchos procesos se limitan a abrir espacios de consulta, como puede observarse en la tabla 6 . Aunque intensidad y calidad técnica no están necesariamente asociadas, en el caso de los proyectos analizados, los procesos de mayor intensidad de participación (de toma de decisiones) son, al mismo tiempo, los de mayor calidad y/o cumplimiento de los objetivos (es decir, los que han llevado a cabo un proceso de participación más completo, ya sea en el modelo integral, complementario, de ordenación o de reglamentación; o un proceso mejor resuelto, en el caso de los programas de apoyo a la participación). Y también son los que han integrado participación a nivel individual en mayor medida.

Además, los procesos de mayor intensidad participativa pertenecen a poblaciones en que en el Ayuntamiento existe un área de participación consolidada, con técnicos contratados (ya sea a tiempo parcial o tiempo completo); y es, asimismo, donde se han dado mayores niveles de transversalidad y donde existen experiencias anteriores en temas de participación. Aunque también se rebela del análisis que es mucho más importante para el desarrollo de un proyecto de mayor intensidad participativa la voluntad política que lo sostiene, que la presencia de profesionales de la participación.

También un número elevado de procesos básicamente de consulta, en los que se usa sobre todo herramientas como las entrevistas y encuestas. Estos procesos suelen estar dirigidos a las asociaciones y a políticos y técnicos. En el caso de poblaciones muy pequeñas se afirma que la consulta se ha realizado a través de visitas «puerta a puerta» o a través de encuentros informales en la calle, el bar, etc., del municipio.

\section{TABLA 6}

Intensidad de la participación

\begin{tabular}{|l|c|c|}
\hline & Frecuencia & Porcentaje \\
\hline Información & 3 & 8,1 \\
\hline Comunicación & 2 & 5,4 \\
\hline Consulta & 9 & 24,3 \\
\hline Deliberación & 8 & 21,6 \\
\hline Participación en decisiones & 11 & 29,7 \\
\hline No corresponde & 4 & 10,8 \\
\hline Total & 37 & $\mathbf{1 0 0 , 0}$ \\
\hline
\end{tabular}

Fuente: Elaboración propia.

${ }^{11}$ Por deliberación entendemos todos aquellos procesos que evalúan y/o priorizan las diferentes propuestas en función de unos criterios previamente definidos de forma conjunta. En la toma de decisiones incluimos aquellos procesos en los que los participantes han definido un plan o proyecto concreto y éste, o bien es de aplicación directa, o sólo le queda ser ratificado por el pleno del Ayuntamiento, sin ser objeto de negociación en ningún otro espació político o público. También incluimos en la toma de decisiones aquellos espacios en que los participantes (entidades e individuos) cogestionan el proceso juntamente con los técnicos y políticos. 
Más allá de los espacios de participación presencial o virtual, también se han realizado en muchos casos campañas de información y difusión. Aunque sólo un $8 \%$ de los proyectos son exclusivamente informativos, la gran mayoría ha realizado en un momento u otro, algún tipo de difusión a través de los canales de comunicación local habituales.

b) Coordinación con el sistema participativo. Consideramos que la posibilidad de coordinación con otros procesos de participación del municipio es una potencialidad positiva para todo proceso o mecanismo participativo. Aunque no se ha contrastado este elemento de forma general a través de las encuestas, se han podido identificar, a través de las entrevistas, algunas reflexiones importantes en relación a este punto.

En el caso de los planes de participación parece identificarse una estrategia específica de ordenación e incluso, en algunos casos, de retroalimentación, con otros procesos participativos existentes, dado que se usan espacios que ya funcionan para debatir y definir los planes de participación. En cambio, en el caso de los programas de apoyo a la participación (urbanismo, asociacionismo, mediación, TIC's, etc.) la coordinación con otros procesos ha sido minoritaria y en muy pocos casos parece encontrarse enmarcada en una estrategia municipal integral de participación.

La coordinación entre los proyectos y los espacios y canales estables de participación (Consejos Consultivos, etc.) es, en general, escasa, principalmente en los programas de apoyo a la participación. Parece que los espacios y canales estables sean estrictamente formales y protocolarios; y que en momentos en que estos espacios puedan jugar un papel más activo en la política local, se acaben abriendo nuevos espacios ad hoc de carácter puntual. En estos casos, a los espacios existentes se les da una función informativa o de presentación en el inicio o en la fase final del proceso.

c) Metodologías empleadas y contratación de expertos. Muy pocas veces se han usado «técnicas innovadoras» de debate. En los casos en que se han aplicado, casi siempre se debe a la presencia de una empresa externa encargada de la gestión del proyecto. Las «técnicas innovadoras» de debate más utilizadas han sido el DAFO, las aportaciones con tarjetas, los talleres de visión de futuro y los talleres inspirados en la metodología EASW u otras técnicas específicas de deliberación y síntesis de la información. En el resto de proyectos, los grupos de trabajo, las reuniones, las comisiones, los consejos, etc., no han empleado ninguna técnica específica o innovadora.

La presencia de empresas externas, pero especialmente la presencia de expertos que gestionen procesos tiene efectos diversos. Por un lado, en general, sirven para introducir metodologías y dinámicas participativas innovadoras y específicas; y su rol de hacer «pedagogía de la participación» a cargos políticos y técnicos de los Ayuntamientos y miembros de asociaciones es de vital importancia. Por otro lado, se puede caer fácilmente en la estandardización del diseño metodológico del proceso, algo que no tiene en cuenta la especificidad y potencialidad genuina de lo local. Según el perfil de población, las contrataciones de empresas externas se dan mayoritariamente en poblaciones grandes o muy grandes en las que el Ayuntamiento tiene un área de participación propia y que necesitan un asesoramiento externo para realizar una tarea de ordenación interna, de formación o de apertura hacia las asociaciones y/o ciudadanía individual. También se da, aunque marginalmente, en poblaciones medianas, con recursos económicos, sin área de participación consolidada y con un técnico responsable de otras áreas. Las poblaciones pequeñas, sin áreas ni técnicos de participación y con presupuestos a menudo menores, acostumbran a contratar un técnico externo o profesional de la participación (no una empresa) para llevar a cabo sus proyectos.

Finalmente, a menudo se afirma que los municipios pequeños tienen una estrategia específica o dinámica particular de participación, pero de las entrevistas realizadas a representantes políticos y técnicos de pequeños municipios se deduce que los diseños o estrategias metodológicas usadas no son distintivas, ni innovadoras respecto municipios de tamaño mayor. Tan sólo, y en algunas ocasiones, se pueden identificar estrategias de información, comunicación o consulta, más personalizadas (visitas nominales, llamadas telefónicas, altavoces callejeros, o encuentros informales en 
la calle, en el bar, etc., del municipio). El hecho de ser una población con pocos habitantes, y con un mismo nivel de esfuerzo, facilita, en estos tres niveles, la proporción de individuos a la que se hace extensivo el proceso.

d) Fases de los procesos de participación. De las diagnosis realizadas en los planes de participación debemos diferenciar aquellas que se han llevado a cabo sólo a nivel interno del Ayuntamiento, de aquellas realizadas externa y participativamente. Las diagnosis internas del Ayuntamiento han consistido generalmente en el análisis de experiencias participativas previas; entrevistas en profundidad a técnicos y políticos del Ayuntamiento; y, en aquellos casos en los que se han empleado metodologías más innovadoras, se han realizado talleres usando estrategias específicas (como el DAFO) para dinamizar el debate de técnicos y políticos por separado. La participación de miembros de asociaciones y de ciudadanos a título individual ha sido prácticamente inexistente, y en los casos en los que se ha dado, se ha vehiculado a través de los Consejos Sectoriales, pero de manera meramente informativa o consultiva.

Las diagnosis externas y participadas son las que han incluido de forma más activa, además de los técnicos y políticos del Ayuntamiento, a miembros de asociaciones y ciudadanos. En estos casos, se ha realizado una diagnosis de los mecanismos de participación existentes y del tejido asociativo del municipio mediante talleres abiertos. A pesar de ello, bajo el término «talleres de diagnosis» se han englobado prácticas muy diversas, más o menos intensivas y abiertas.

El número de procesos que han alcanzado fases de deliberación y diseño de propuestas ha sido menor que los de diagnosis; es necesario tener en cuenta que muchos planes de participación habían pedido sólo subvención para la primera parte del proceso. Volvemos a diferenciar dos tipos de proyectos. Primero, aquellos en que la deliberación se ha llevado a cabo a nivel interno: en este caso no se ha especificado como eran esos espacios de deliberación (llamados a menudo »mesas de coordinación» 0 «comisiones de participación») o la información que se da es a menudo muy confusa. Segundo, aquellos en que los espacios de deliberación se han abierto a las asociaciones y la ciudadanía individual ${ }^{12}$. En muy pocos casos se han aplicado estrategias específicas para incluir la participación de ciudadanos a título individual; y en algún proceso en concreto han participado exclusivamente asociaciones. Minoritariamente los resultados del espacio de deliberación se han completado a través de entrevistas, cuestionarios y propuestas recogidas a través de oficinas de información, forum electrónicos y buzones públicos.

La fase de toma de decisiones se puede dar a lo largo de todo el proceso a través de una cogestión del mismo, dándose marginalmente; normalmente se consolida formalmente a través de la ratificación y aprobación de las decisiones tomadas durante el proceso participativo en el pleno municipal. Los procesos aprobados por el pleno son los que cualitativamente han tenido un mejor desarrollo.

Finalmente, cabe destacar la escasa existencia de fases de seguimiento y devolución de los resultados en los procesos estudiados, independientemente de si se trataba planes o programas.

\subsubsection{Resultados obtenidos}

Si nos centramos en los resultados relacionales podemos ver que prácticamente todos los planes de participación, según afirman los responsables, han contribuido a mejorar y aumentar la comunicación y la transversalidad interna a nivel políitco, técnico y entre estos dos niveles, así como también a entrar en contacto y/o «mover» el tejido asociativo del municipio. En general, el contacto con la ciudadanía no se ha visto fortalecido.

${ }^{12}$ Nos referimos a: talleres y jornadas de debate y deliberación; sesiones de trabajo; Consejos Sectoriales; consejos más generalistas como Consejos Abiertos, Consejos Populares; Consejos Territoriales; comisiones específicas creadas únicamente para el proceso; etc. 
En el caso de los programas de apoyo a la participación no se afirma con tanta contundencia que el proceso haya contribuido a mejorar y aumentar las relaciones internas, siendo esto más relevante para aquellos proyectos impulsados desde un área específica del Ayuntamiento sin conexión con el resto y en Gobiernos de coalición.

Tanto de las entrevistas como de las encuestas realizadas se desprende que, en general, se tiende a sobredimensionar o sobrevalorar el efecto positivo del proceso en las relaciones entre los diferentes actores.

Por lo que se refiere a los resultados substantivos, estos son desiguales: 1) En más de la mitad de los casos, el proceso ha servido para concretar un reglamento o un plan con previsión de acciones concretas. 2) En otros, las conclusiones del proceso se resume en una de diagnosis y algunas reflexiones sobre posibles estrategias o líneas de trabajo futuras, pero sin acciones concretas a desarrollar. 3) E incluso una parte importante de casos el proyecto estaba en desarrollo en el momento de la evaluación ${ }^{13}$ y, por tanto, sólo se dispone de los resultados de la diagnosis.

En la mayoría de los casos en los que se concretan resultados propositivos, estos se encaminan fundamentalmente a sistematizar canales y acciones para tener un control más centralizado de la participación. Pero es específicamente en el desarrollo de reglamentos de participación dónde esta tendencia formalizadora y centralizadora se da en mayor medida.

En los programas de apoyo a la participación los resultados han sido más diversos que en los planes, concentrando los primeros un mayor número de experiencias fallidas (subvenciones devueltas, objetivos no alcanzados, procesos a medio realizar, menor intensidad participativa, etc.).

\subsubsection{Evaluación del proceso}

Por lo que atañe a la evaluación del proceso de participación, en general no se tiene en cuenta ni se lleva a cabo. A pesar de todo, podemos identificar diferentes situaciones:

En la mayoría de casos, aún estando prevista en el proyecto (seguramente porque éste era uno de los requisitos de la convocatoria de la DGPC para planes de participación), no se ha implementado. En los programas de apoyo a la participación, para los cuales la DGPC no especificaba la evolución como requisito, en general, ésta ni se planteaba su diseño.

En los pocos casos que llevan a cabo una evaluación, suele estar sistematizada vía encuesta, con o sin complemento de evaluación cualitativa. Son evaluaciones realizadas por empresas externas respeto una parte concreta del proceso (evaluaciones parciales) y se centran básicamente, aunque no de manera exclusiva, en el grado de satisfacción de los participantes en el proceso y en el funcionamiento de ese espacio concreto de participación que se está evaluando, sin tener en cuenta, la mayoría de las veces, los resultados substantivos obtenidos.

A nivel interno, en algunos casos, se han hecho valoraciones poco sistemáticas del proceso, que no evaluaciones, en el marco de reuniones entre técnicos y políticos que gestionan y/o lideran el proceso (comisiones de seguimiento, grupo promotor, comisión de participación, etc.)

En pocos casos se han hecho evaluaciones continuas a lo largo del proceso en los diferentes espacios de participación y con diferentes actores.

\footnotetext{
${ }^{13}$ La recogida de datos de la evaluación se cierra el 31 de marzo de 2006.
} 


\section{Conclusiones}

Del análisis anterior podemos extraer unas conclusiones y aprendizajes generales:

- Se da una concentración de solicitudes en el área metropolitana. Las causas son diversas, pero a medida que las formas participativas y el debate en torno a esta cuestión se van extendiendo, se supone que esta tendencia se irá suavizando. Así, la gran potencialidad que se observó en la convocatoria del 2005, a saber, la presentación de solicitudes por parte de entes sin experiencia previa en procesos de participación, se está viendo corroborada e intensificada en la convocatoria del 2006.

- Las temáticas para las que se piden las subvenciones son variadas y no dependen de la zona geográfica, pero se constata que hay pocos planes de participación y planes estratégicos y, en cambio, muchos proyectos de carácter urbanístico. También hay bastantes proyectos relativos a la convivencia, la mediación y la inmigración, algo que nos lleva pensar que los procesos participativos se entienden a menudo como una buena herramienta para la gestión comunitaria y de resolución los conflictos interculturales cada vez más habituales en nuestra sociedad.

- Cabe preguntarse hasta que punto la planificación de la participación se puede extender más allá de la necesidad de ordenar procesos realizados con anterioridad: aunque idealmente la planificación estratégica es un paso previo a todo desarrollo participativo, los entes sin experiencia previa han fracasado en el abordaje integral de la participación. En caso de valorarse la planificación estratégica positivamente, seria necesario un apoyo técnico externo que supliera la falta de experiencia.

Respeto a los municipios analizados, apuntamos algunos elementos de reflexión interesantes sobre la naturaleza de la participación, la estructura de un proceso y los resultados esperados. Los elementos son los siguientes:

- Concepciones de la participación: Se constata, que hay concepciones muy diferentes de la participación ciudadana y esto se concreta en modelos y estrategias de desarrollo y aplicación también muy diferentes, con mayor o menor grado de implicación de los participantes y políticos y con una centralidad variable del proyecto en el marco de la acción de gobierno. En cualquier caso, es importante saber de qué modelo se parte, principalmente para tener claros los principios que regirán el proceso participativo.

- Participantes: Se da una débil incorporación de nuevos participantes, ya que las experiencias se dirigen, en general, a los «interlocutores habituales». Paralelamente, hay poco control de la diversidad de los participantes, algo que dificulta la detección e incorporación de colectivos con menor presencia en los mecanismos de participación tradicionales (jóvenes, inmigrantes, etc.). Aunque a priori resulte más sencillo la relación con ciudadanos y asociaciones habituales, los procesos participativos pierden potencialidad y capacidad de transformación social si se limitan a este tipo de actores. En este sentido, se constata que se usa poco el recurso de los grupos promotores en que son los propios ciudadanos habituales los que llevan a cabo una tarea de movilización de personas generalmente pasivas o no encuadradas en ninguna asociación, las cuales suelen aceptar mejor ser convocadas por amigos y vecinos que no por cualquier Administración. Finalmente, se suele potenciar poco la participación interna en las asociaciones, algo que también favorecería la representación real de los presidentes de las asociaciones y se incorporarían en el diálogo muchas más voces y puntos de vista, aunque no se hallasen presentes en el momento del debate presencial. 
- Transversalidad: La implicación de áreas y departamentos distintos a los que toman la iniciativa tiende a ser baja, aunque los procesos ayuden a consolidar relaciones y en algún caso a fortalecerlas. Pero en general, se tiende a sobrevalorar el efecto positivo del proceso en las relaciones entre los diferentes actores y la transversalidad interna en los entes locales. Esto nos lleva a pensar que, aunque se conciba la participación como una herramienta de gobernación importante en el ámbito local, ésta tiene un rol de complemento, no estructural.

- Metodología y resultados: Tanto el grado de innovación en las metodologías empleadas, como los resultados obtenidos han sido desiguales. Algunas causas importantes de innovación y éxito son la presencia de áreas técnicas específicas, el bagaje anterior en experiencias de participación ciudadana y la centralidad política que se atribuye al proyecto. Otras causas tienen que ver con las características de la convocatoria de la DGPC, especialmente aquellos aspectos que se refieren a los límites temporales y económicos estipulados en la convocatoria. Por otro lado, la presencia de profesionales de la participación contratados para conducir técnicamente el proceso, favorece un desarrollo más ordenado y sistemático de los proyectos.

- Evaluación: La falta de evaluación de los procesos participativos es considerable en los casos analizados. Este hecho refleja que, a pesar de apostar por la innovación democrática, los criterios en los que esta innovación se basa, no están siempre claros o explicitados, con lo que no queda bien definida la frontera entre los criterios políticos y los criterios técnicos en la definición y desarrollo del proyecto. Seguramente, los puntos anteriores y las potencialidades de los diferentes municipios y comarcas en relación a la participación mejorarían substancialmente si los procesos de evaluación, y especialmente los de autoevaluación se llevaran a cabo.

Con todo, aunque que con debilidades, los procesos analizados demuestran la extensión y diversidad de estas herramientas a nivel local. Se detectan potencialidades y oportunidades importantes, como hemos visto a lo largo del análisis.

\section{REFERENCIAS BIBLIOGRÁFICAS Y DOCUMENTALES}

Anduiza, E. y de Maya, S. (2005), La qualitat de la participació: una proposta d'indicadors. Barcelona: Fundaciò J. Bofill.

Arnstein, S. (1971), «A Ladder of participation in the USA», Journal of the Royal Town Planning Institute 57: 176-182.

Blanco, I. y Gomà. R. coords., (2002), Gobiernos Locales y Redes Participativas. Barcelona: Ariel.

Delbecq, A. L. et. al., (1984), Técnicas grupales para la planeación. México DF: Trillas.

Font, J. coord., (2001), Ciudadanos y decisiones públicas. Barcelona: Ariel.

Font, J. y Blanco, I. (2003), Experiències de participació ciutadana. Polis, la ciutat participativa. Participar en els municipis: qui, com i per què? Barcelona: Centre per a la Participació Ciutadana, Diputació de Barcelona.

Fundación Kaleidos red (2006), Manual de equipamientos municipales de proximidad. Planificación constructiva, perfiles profesionales y participación ciudadana. Madrid: Imagine Ed.

Jorba, L.; Martí, J. y Parés, M. (próxima publicación), Processos de participació ciutadana: orientacions per a l'avaluació participada. Barcelona: Fundació Bofill. 
Lorenzana, C. ed., (2002), Tomamos la palabra. Experiencias de ciudadanía participativa. Barcelona: Icaria/ACSUR-Las Segovias.

Marchioni, M. (1999), Comunidad, participación y desarrollo. Teoría y metodología de la intervención comunitaria. Madrid: Popular

Merino, M. coord., (1994), En busca de la democracia municipal. La participación ciudadana en el gobierno local mexicano. México DF: El Colegio de México.

Pindado, F. (2000), La participación ciudadana en la vida de las ciudades. Barcelona: Ediciones del Serbal.

Putnam, R. D. (1993), Per fer que la democràcia funcioni. La importància del capital social. Barcelona: Proa.

Rebollo, O. y Martí, J. (2002), Eines per a la participació ciutadana. Bases, mètodes i tècniques. Barcelona: Diputació de Barcelona.

Subirats, J. et al. (2001), Experiències de participació ciutadana en els municipis catalans. Barcelona: Escola d'Administració Pública de la Generalitat de Catalunya.

Villasante T. R., Montañés, M. y Martí. J. coords., (2000), La Investigación social participativa. Construyendo ciudadanía 1. Barcelona: El Viejo Topo/Red CIMS.

Villasante T. R., Montañés, M. y Martí. J. coords., (2001), Prácticas locales de cratividad social. Construyendo ciudadanía 2. Barcelona: El Viejo Topo/Red CIMS.

Villasante, T. R. (1995), Las democracias participativas. De la participación ciudadana a las alternativas de sociedad. Madrid: HOAC. 